
\title{
25 Research Suare \\ Infarct Growth Precedes Cerebral Thrombosis Following Experimental Stroke in Mice
}

\author{
Vanessa Göb \\ University Hospital Würzburg \\ Maximilian G. Voll \\ University Hospital Würzburg \\ Lena Zimmermann \\ University Hospital Würzburg \\ Katherina Hemmen \\ University of Würzburg \\ Guido Stoll \\ University Hospital Würzburg \\ Bernhard Nieswandt \\ University Hospital Würzburg \\ Michael K. Schuhmann \\ University Hospital Würzburg \\ Katrin G. Heinze \\ University of Würzburg \\ David Stegner ( $\square$ stegner@virchow.uni-wuerzburg.de ) \\ University Hospital Würzburg
}

\section{Research Article}

Keywords: ischemic stroke, thrombosis, platelet, reperfusion injury

Posted Date: November 1st, 2021

DOI: https://doi.org/10.21203/rs.3.rs-731818/v1

License: (c) (1) This work is licensed under a Creative Commons Attribution 4.0 International License.

Read Full License

Version of Record: A version of this preprint was published at Scientific Reports on November 24th, 2021. See the published version at https://doi.org/10.1038/s41598-021-02360-6. 


\section{Abstract}

Ischemic stroke is among the leading causes of disability and death worldwide. In acute ischemic stroke, successful recanalization of occluded vessels is the primary therapeutic aim, but even if it is achieved, not all patients benefit. Although blockade of platelet aggregation did not prevent infarct progression, cerebral thrombosis as cause of secondary infarct growth has remained a matter of debate. As cerebral thrombi are frequently observed after experimental stroke, a thrombus-induced impairment of the brain microcirculation is considered to contribute to tissue damage. Here, we combine the model of transient middle cerebral artery occlusion (tMCAO) with light sheet fluorescence microscopy and immunohistochemistry of brain slices to investigate the kinetics of thrombus formation and infarct progression. Our data reveal that tissue damage already peaks after $8 \mathrm{~h}$ of reperfusion following $60 \mathrm{~min}$ MCAO, while cerebral thrombi are only observed at later time points. Thus, cerebral thrombosis is not causative for secondary infarct growth during ischemic stroke.

\section{Introduction}

Stroke is the second leading cause of death and disability worldwide and mostly caused by thromboembolic occlusion of major brain vessels. In human stroke the middle cerebral artery (MCA) and its branches are most commonly affected and account for approximately $70 \%$ of infarcts ${ }^{1}$. There are several models available to study ischemic stroke in animals. The model of transient middle cerebral artery occlusion ( $\mathrm{MCAO}$ ) affects the MCA territory and is very close to the human situation and therefore widely used in experimental stroke research ${ }^{2,3}$. For the therapy of acute ischemic stroke, recanalization of the occluded vessel is essential, but not sufficient to guarantee brain salvage ${ }^{4}$. The phenomenon of ongoing ischemic lesion development despite recanalization is referred to as reperfusion injury. Often, abnormalities in cerebral blood perfusion lead to unfavorable outcomes as post-stroke hypoperfusion is associated with infarct expansion ${ }^{5}$. In the tMCAO model, complete recanalization is achieved by the removal of the filament, however cerebral perfusion is still not restored to $100 \%$ but only to approximately $50 \%{ }^{6}$. It is well known that platelets contribute to ischemia/reperfusion injury of the brain ${ }^{7}$, but it is still controversially discussed whether ongoing tissue damage is caused by cerebral microthrombosis or not. Thrombotic activity after recanalization is still widely seen as one cause for the breakdown of the microcirculation, incomplete reperfusion and subsequent tissue death ${ }^{8-10}$. However, the fact that complete blockade of platelet aggregation via anti-GPIlb/Illa treatment was not beneficial in either mice ${ }^{11}$ or patients ${ }^{12,13}$, suggests that thrombus formation is an unlike reason for tissue death. To clarify this, we examined the time course of infarct progression, platelet deposition and thrombus formation in the murine brain after IMCAO and confirmed that the occurrence of cerebral thrombi correlated with infarct size after $24 \mathrm{~h}$ but was secondary to neuronal damage.

\section{Methods}

\section{Animals}


Animal experiments were performed following the regulations of the National Institute of Heath Guidelines for the Care and Use of Laboratory Animals in compliance with ARRIVE guidelines ${ }^{14}$ and the approval of local authorities (District government of Lower Franconia). To reduce variability and thereby mouse numbers, only male mice with an age of 10-14 weeks were used. Recent studies showed remarkable effects of sex differences on the inflammatory response and infarct sizes ${ }^{15}$. Assuming a reduction of of $30 \%$ as functionally relevant and a standard deviation of $20 \%$ to the respective mean values, a group size of $\geq 8$ was necessary to show this effect with a power of 0.8 and a probability of a type I error of $<0.5$ (calculated with GraphPad StatMate 2.00). The distribution of the mice into the different groups was randomized using research randomizer (randomizer.org).

\section{Transient middle cerebral artery occlusion}

10- to 14-week-old, male, C57BL/6J mice were applied to the tMCAO model and infarct sizes were determined as previously described ${ }^{6}$. Mice were anesthetized by $2 \%$ isoflurane inhalation anesthesia. A silicon rubber-coated filament (6021PK10, Doccol, Redlands, CA) was inserted in the carotid artery and advanced up to the origin of the middle cerebral artery (MCA). Surgical procedure was kept below 10 min for each mouse. After $60 \mathrm{~min}$, the filament was removed allowing reperfusion. Animals were sacrificed after the indicated reperfusion times and brains were removed for further analysis. Exclusion criteria were death of the animals following tMCAO or signs of subarachnoidal hemorrhage (SAH) noted during brain sampling.

\section{Infarct size measurement}

Removed brains were cut into three $2 \mathrm{~mm}$ thick coronal sections and viable tissue was stained with $2 \%$ 2,3,5 triphenyltetrazolium chloride (TTC; Sigma-Aldrich) for $20 \mathrm{~min}$ at $37^{\circ} \mathrm{C}$. Brain slices were scanned, and edema-corrected infarct volumes were calculated by planimetry (ImageJ Software, National Institutes of Health) with the following equation: $V_{\text {indirect }}\left(\mathrm{mm}^{3}\right)=\mathrm{V}_{\text {infarct }} \mathrm{x}(1-(\mathrm{VI}-\mathrm{VC}) / \mathrm{VC})$. VI: Volume ischemic hemisphere, VC: Volume control hemisphere.

\section{Immunohistochemistry}

After staining of infarct volumes, the middle brain segment was cryo-embedded and cut into $10 \mu \mathrm{m}$ thick slices. For the quantification of occluded vessels, brain slices were stained with hematoxylin and eosin. The number of occluded and open vessels was counted from the blinded samples under 20-fold magnification in the ischemic hemisphere and the percentage of occluded vessels was calculated.

For quantification of the number of platelet rich thrombi, platelets were stained with AF594-conjugated anti-platelet GPIX derivative ${ }^{16}(5 \mu \mathrm{g} / \mathrm{ml})$; nuclei were stained with DAPI. Sections were imaged using a Leica SP8 confocal microscope and the whole brain section was recorded. Thrombi were quantified from 
tile scans using the Analyze Particle tool in Fiji (ImageJ Software, National Institutes of Health). Every "particle" larger than $5 \mu \mathrm{m}^{2}$ was counted as thrombus.

\section{Western Blot}

Protein extraction and Western blot analysis were performed according to standard procedures and as previously described ${ }^{17}$. Dissected cortices were homogenized in RIPA buffer containing protease inhibitor cocktail (cOmplete ${ }^{\mathrm{TM}}$ protease inhibitor cocktail, Sigma-Aldrich) and sonified for $10 \mathrm{sec}$. Residual tissue was removed via centrifugation at $15.000 \mathrm{~g}$ for $30 \mathrm{~min}$ at $4^{\circ} \mathrm{C}$. After determination of the protein content in the supernatant via bicinchoninic acid (BCA), lysates were used for Western Blot analysis. Anti-mouse GPIb $\beta$ antibody p0p $1^{18}(10 \mu \mathrm{g} / \mathrm{ml})$ was used for the quantification of platelet protein in the brain tissue. Actin (anti-Actin, A5441, Merck; 1:250,000) was used as loading control and for normalization of protein content.

\section{Light sheet fluorescence microscopy (LSFM)}

$30 \mathrm{~min}$ before the end of the desired reperfusion time platelets and the vasculature were stained by intravenous injection of AF750-conjugated anti-GPIX derivative $(0.6 \mu \mathrm{g} / \mathrm{g} \text { body weight })^{19}$, AF647conjugated anti-CD105 ${ }^{20}$ and AF647-conjugated anti-CD31 (each $0.4 \mu \mathrm{g} / \mathrm{g}$ body weight; BioLegend). 30 min after in vivo labeling (= indicated reperfusion times), mice were anesthetized by intraperitoneal injection of medetomidine $0.5 \mu \mathrm{g} / \mathrm{g}$, midazolam $5 \mu \mathrm{g} / \mathrm{g}$ and fentanyl $0.05 \mu \mathrm{g} / \mathrm{g}$ body weight and transcardially perfused with ice-cold PBS and ice-cold 4\% paraformaldehyde (PFA, Sigma-Aldrich, Schnelldorf, Germany, pH 7.2). Brains were removed, dehydrated in methanol solutions of increasing concentrations $(50 \%, 70 \%, 95 \%, 100 \%)$ for $8-12 \mathrm{~h}$ each at $4^{\circ} \mathrm{C}$ and stored in $100 \%$ methanol overnight. For optical clearing, brains were incubated in BABB solution (2 parts benzyl benzoate mixed with 1 part benzyl alcohol; both Sigma-Aldrich) for $12 \mathrm{~h}$ and stored in fresh BABB solution for another $12 \mathrm{~h}$ before imaging. Optically cleared brains were imaged with a custom-build light sheet microscope equipped with two EC Epiplan. Neofluar 2.5x/0.06 M27 excitation objectives (Zeiss, Germany) and a HCX FLUOTAR $5 x / 0.15$ Dry detection objective (Leica, Germany) as previously described ${ }^{19}$. Information about the analysis of the LSFM images is given in the supplemental methods.

\section{Statistical analysis}

Normal distribution of the data was analyzed using D'Agostino \& Pearson omnibus normality test. Since normality was not met by all subsets, statistical differences between two groups were analyzed using the non-parametric two-tailed Mann-Whitney $U$ test. P-values $<0.05$ were considered statistically significant and $p$-values are indicated in the figures. In all figures, results are indicated as mean \pm SD. 


\section{Results}

In our attempt to investigate the correlation of cerebral thrombosis and infarct progression we subjected mice to the IMCAO model with a standard occlusion time of $60 \mathrm{~min}$ and characterized the time course of infarct growth. After different reperfusion times, infarct sizes were determined. Infarcted tissue (white) can be seen first after $4 \mathrm{~h}$ of reperfusion (Fig. 1A). At this time point individual variance is the highest, as some mice show already large infarcts, whereas others do not (Fig. 1, 2nd and 3rd panel, Fig. 1B). After 8 $\mathrm{h}$ of reperfusion, however, infarcts have reached their maximum size $\left(122.4 \pm 29.65 \mathrm{~mm}^{3}\right)$ and do not further increase with longer reperfusion time (infarct size after $24 \mathrm{~h}: 124.0 \pm 18.67 \mathrm{~mm}^{3}$; Fig. 1A,B) indicating that processes leading to tissue death must occur within the first $8 \mathrm{~h}$ of the reperfusion phase.

To analyze the formation of thrombi in the time course of reperfusion we analyzed whole brain hemispheres in 3D using light sheet microscopy and developed an image processing and analysis strategy to quantify thrombi within the $3 \mathrm{D}$ reconstructions. In brains of sham-operated mice, almost no thrombi could be detected ( $25 \pm 18$; Fig. 1C, Suppl. Video 1). In brains that were removed after $4 \mathrm{~h}$ or $8 \mathrm{~h}$ of reperfusion, no significant increase in thrombus number was detected ( $98 \pm 86$ and $61 \pm 25$, respectively; Fig. 1D,E; Suppl. Videos 2, 3). Quantifying thrombus number in brains that were removed after $24 \mathrm{~h}$ of reperfusion revealed significantly elevated numbers ( $468 \pm 463$; Fig. 1F, Suppl. Video 4 ) and a clear difference can be seen in comparison to brains after $8 \mathrm{~h}$ of reperfusion, despite comparable infarct sizes (Fig. 1B). These data indicate that tissue death following experimental stroke precedes cerebral thrombus formation.

To exclude that the lack of cerebral thrombi is a result of perfusion artefacts during sample preparation for light sheet microscopy, we analyzed cryosections of brain segments that were removed at the indicated time points without prior perfusion fixation. We quantified the number of occluded vessels in hematoxylin and eosin-stained sections (Fig. 2A) and the number of thrombi in sections stained specifically with an anti-platelet antibody (Fig. 2B,C). After $24 \mathrm{~h}$ of reperfusion, the percentage of occluded vessels and the number of thrombi (36.83 $\pm 7.46 \%$ and $261.5 \pm 115.4$, respectively) are significantly higher than for sham operated mice $(22.14 \pm 8.59 \%$ and $89.74 \pm 84.05)$ which correlates with the infarct sizes (Fig. 1B). Strikingly, at earlier time points, after $4 \mathrm{~h}$ and after $8 \mathrm{~h}$ of reperfusion, the number of occluded vessels ( $4 \mathrm{~h}: 21.29 \pm 8.95 \%$; $8 \mathrm{~h}: 24.29 \pm 7.86 \%$ ) as well as the number of thrombi (4 h: $30.89 \pm 25.67$; 8 h: $20.29 \pm 19.45$ ) were comparable to those of sham operated mice (Fig. 2A-C). Given the fact that infarct sizes have already reached their maximum after $8 \mathrm{~h}$, the absence of thrombi at this time point indicates that cerebral microthrombi are not causative for infarct progression.

For an imaging independent quantification of platelets within the brain hemispheres, we performed Western Blot analysis of brain tissue and quantified the amount of GPIb $\beta$, a platelet-specific protein (that is not released from activated platelets), within the cortical region of the brains. Here, the relative GPIb $\beta$ content in brain tissue of sham operated mice $(0.81 \pm 0.11)$, after $4 \mathrm{~h}(1.07 \pm 0.55)$ or $8 \mathrm{~h}(1.15 \pm 0.57)$ of reperfusion is similar and only after $24 \mathrm{~h}$ of reperfusion, GPIb $\beta$ content is significantly increased (2.39 \pm 
1.05; Fig. 2D). The contralateral hemisphere was taken as internal control and shows no increase in GPIb $\beta$ levels at any time point.

\section{Discussion}

The primary therapeutic goal for acute ischemic stroke patients is the recanalization of the occluded vessel allowing reperfusion of the ischemic tissue. Despite restoration of blood flow, not all patients benefit, and further ('secondary') infarct growth can be observed ${ }^{4}$. The degree of cerebral perfusion after recanalization has been found to correlate with infarct progression ${ }^{21}$. Thrombi in the microcirculation, that either result from embolization of the initial stroke causing occlusion (during reperfusion) or emerge secondary within the cerebral vasculature, would be a plausible explanation for the insufficient reperfusion. In line with this, several studies showed a positive correlation of the occurrence of occluded vessels/thrombi with infarct sizes at day 1 after $\mathrm{tMCAO}^{22-24}$. However, interpretation of these data must be taken with care because this does not necessarily mean that vessel occlusion is the reason for infarct development. Nevertheless, these results have spurred the concept that thrombotic events during reperfusion trigger a breakdown of the microcirculation resulting in tissue damage and secondary infarct growth.

We have investigated the kinetics of infarct progression following experimental stroke and already after 4 $\mathrm{h}$ of reperfusion significant infarcts are developing. At this early time point after $\mathrm{tMCAO}$ the infarct sizes are variable (Fig. 1B), indicating that the infarct growth is not complete, while at $8 \mathrm{~h}$ and $24 \mathrm{~h}$, all mice have big infarcts and no further increase in infarct size was observed between 8 and $24 \mathrm{~h}$. Using several complementary approaches, we reveal here that thrombi can only be detected in the cerebral vasculature after infarct progression excluding thrombosis as cause for secondary infarct growth. We analyzed the number of occluded vessels using H\&E staining (Fig. 2A) as surrogate marker for cerebral thrombosis and determined cerebral thrombi via immune fluorescence staining (Fig. 2B,C). In both cases, thrombi were also observed in the sham group, indicating limitations of those two methods, as some residual platelet activity might be triggered by the death of the animals. Furthermore, thin sections have the limitation of providing few information in z-plane. In contrast to this, Western blotting (Fig. 2D), where normalization to the contralateral hemisphere and LSFM, which includes perfusion of the animals, have less 'thrombotic activity' in the sham group and provide information of the situation in the whole hemisphere. Notably, however, independently of the methodology used no differences were observed between the sham group and the $4 \mathrm{~h}$ and $8 \mathrm{~h}$ reperfusion groups, while $24 \mathrm{~h}$ after reperfusion significantly more thrombi were observed.

The occurrence of thrombi in the infarcted brain tissue after tissue damage might play a dual role. On the one hand, thrombi might further decrease supply of the infarcted tissue and promote later stages of tissue death. On the other hand, thrombi are likely the consequence of ongoing tissue death in the infarcted brain areas. Tissue death liberates platelet activating mediators which promote thrombus formation in the affected areas. Upon activation, platelets release a variety of factors that are known to be involved in angiogenesis, immune-modulation and tissue repair ${ }^{25}$. Therefore, thrombus formation 
might be an active part of tissue regeneration. Further studies are warranted to investigate the role of platelets in tissue regeneration after stroke.

In conclusion, our data exclude cerebral thrombus formation as major cause for infarct progression during the reperfusion phase following IMCAO. Of note, these data are in line with the fact that blocking platelet aggregation by targeting GPIIb/IIla failed in stroke therapy ${ }^{12,13}$.

Nevertheless, platelets contribute to reperfusion injury since absence of other platelet receptors or components has been shown to improve outcomes following $\mathrm{tMCAO}^{7}$. Further, blocking initial platelet adhesion improves cerebral perfusion and reduces infarct sizes following experimental stroke ${ }^{26}$ and reduces tissue damage already during the occlusion phase ${ }^{27}$. This supports the concept, that the platelets' role to infarct progression is different from 'just' thrombosis and evidence is given that this might be rather of immune-modulating nature ${ }^{6,7,28}$. Reperfusion injury is a complex phenomenon involving presumably a plethora of cells and molecules (reviewed in ${ }^{29}$ ). Collapse of the microcirculation can have various reasons, it might result from extrinsic compression and/or intravascular events ${ }^{30}$. So far, involvement of astrocytes, pericytes, endothelial cells, leukocytes and small molecules like nitric oxide or acetylcholine has been shown ${ }^{31}$. Platelets might directly contribute to tissue damage, or, potentially, recruit neutrophils, that have been shown to contribute to the no-reflow following ischemic stroke ${ }^{32,33}$. Clearly, future studies are needed to elucidate step by step how platelets and other cell types contribute to the ongoing tissue damage following ischemic stroke.

\section{Declarations}

\section{Data Availability:}

Any material/datasets generated in this study are available upon reasonable request from the corresponding authors.

\section{Funding:}

This work was supported by the Deutsche Forschungsgemeinschaft (SFB/TR 240, project number 374031971, B06 to D.S., M.K.S., K.G.H).

\section{Competing interests:}

The authors declare no competing interests.

\section{Acknowledgements}


The authors would like to thank Ewa Stepien-Bötsch and Oğuzhan Angay for excellent technical assistance.

\section{Authorship Contributions}

Contribution: GS, MKS, DS and KGH designed the study. VG, MGV and LZ performed the experiments. MGV, KGH, KH analyzed the LSFM data. VG, LZ analyzed stainings and WB data. MKS, DS, GS, BN and $\mathrm{KGH}$ discussed results and provided scientific input throughout the study. VG and DS wrote the paper with input and approval from all authors.

\section{References}

1. Bogousslavsky, J., Van Melle, G. \& Regli, F. The Lausanne Stroke Registry: analysis of 1,000 consecutive patients with first stroke. Stroke 19, 1083-1092, doi:10.1161/01.str.19.9.1083 (1988).

2. Fluri, F., Schuhmann, M. K. \& Kleinschnitz, C. Animal models of ischemic stroke and their application in clinical research. Drug Des Devel Ther 9, 3445-3454, doi:10.2147/DDDT.S56071 (2015).

3. Howells, D. W. et al. Different strokes for different folks: the rich diversity of animal models of focal cerebral ischemia. J Cereb Blood Flow Metab 30, 1412-1431, doi:10.1038/jcbfm.2010.66 (2010).

4. Zhu, Z.et al. Combination of the Immune Modulator Fingolimod With Alteplase in Acute Ischemic Stroke: A Pilot Trial. Circulation 132, 1104-1112, doi:10.1161/CIRCULATIONAHA.115.016371 (2015).

5. Zhang, Z., Pu, Y., Mi, D. \& Liu, L. Cerebral Hemodynamic Evaluation After Cerebral Recanalization Therapy for Acute Ischemic Stroke. Front Neuro/ 10, 719, doi:10.3389/fneur.2019.00719 (2019).

6. Schuhmann, M. K.et al. CD84 Links T Cell and Platelet Activity in Cerebral Thrombo-Inflammation in Acute Stroke. Circ Res 127, 1023-1035, doi:10.1161/CIRCRESAHA.120.316655 (2020).

7. Stegner, D., Klaus, V. \& Nieswandt, B. Platelets as Modulators of Cerebral Ischemia/Reperfusion Injury. Front Immuno/ 10, 2505, doi:10.3389/fimmu.2019.02505 (2019).

8. De Meyer, S. F.et al. Thromboinflammation in Stroke Brain Damage. Stroke 47, 1165-1172, doi:10.1161/STROKEAHA.115.011238 (2016).

9. De Meyer, S. F., Stoll, G., Wagner, D. D. \& Kleinschnitz, C. von Willebrand factor: an emerging target in stroke therapy. Stroke 43, 599-606, doi:10.1161/STROKEAHA.111.628867 (2012).

10. Denorme, F.et al. The von Willebrand Factor A1 domain mediates thromboinflammation, aggravating ischemic stroke outcome in mice. Haematologica, doi:10.3324/haematol.2019.241042 (2020).

11. Kleinschnitz, C.et al. Targeting platelets in acute experimental stroke: impact of glycoprotein Ib, VI, and Ilb/Illa blockade on infarct size, functional outcome, and intracranial bleeding. Circulation 115, 2323-2330, doi:10.1161/CIRCULATIONAHA.107.691279 (2007).

12. Adams, H. P., Jr.et al. Emergency administration of abciximab for treatment of patients with acute ischemic stroke: results of an international phase III trial: Abciximab in Emergency Treatment of Stroke Trial (AbESTT-II). Stroke 39, 87-99, doi:10.1161/STROKEAHA.106.476648 (2008). 
13. Kellert, L.et al. Endovascular stroke therapy: tirofiban is associated with risk of fatal intracerebral hemorrhage and poor outcome. Stroke 44, 1453-1455, doi:10.1161/STROKEAHA.111.000502 (2013).

14. Percie du Sert, N.et al. The ARRIVE guidelines 2.0: updated guidelines for reporting animal research. $J$ Physio/ 598, 3793-3801, doi:10.1113/JP280389 (2020).

15. Seifert, H. A.et al. Sex differences in regulatory cells in experimental stroke. Cell Immuno/ 318, 49-54, doi:10.1016/j.cellimm.2017.06.003 (2017).

16. Nieswandt, B., Bergmeier, W., Rackebrandt, K., Gessner, J. E. \& Zirngibl, H. Identification of critical antigen-specific mechanisms in the development of immune thrombocytopenic purpura in mice. Blood 96, 2520-2527 (2000).

17. Franke, M.et al. The NLRP3 inflammasome drives inflammation in ischemia/reperfusion injury after transient middle cerebral artery occlusion in mice. Brain Behav Immun, doi:10.1016/j.bbi.2020.12.009 (2020).

18. Bergmeier, W., Rackebrandt, K., Schroder, W., Zirngibl, H. \& Nieswandt, B. Structural and functional characterization of the mouse von Willebrand factor receptor GPIb-IX with novel monoclonal antibodies. Blood 95, 886-893 (2000).

19. Stegner, D.et al. Thrombopoiesis is spatially regulated by the bone marrow vasculature. Nat Commun 8, 127, doi:10.1038/s41467-017-00201-7 (2017).

20. Gougos, A. \& Letarte, M. Identification of a human endothelial cell antigen with monoclonal antibody 44G4 produced against a pre-B leukemic cell line. J Immunol 141, 1925-1933 (1988).

21. Demeestere, J., Wouters, A., Christensen, S., Lemmens, R. \& Lansberg, M. G. Review of Perfusion Imaging in Acute Ischemic Stroke: From Time to Tissue. Stroke 51, 1017-1024, doi:10.1161/STROKEAHA.119.028337 (2020).

22. Langhauser, F.et al. Kininogen deficiency protects from ischemic neurodegeneration in mice by reducing thrombosis, blood-brain barrier damage, and inflammation. Blood 120, 4082-4092, doi:10.1182/blood-2012-06-440057 (2012).

23. Dittmeier, M., Kraft, P., Schuhmann, M. K., Fluri, F. \& Kleinschnitz, C. Pretreatment with rivaroxaban attenuates stroke severity in rats by a dual antithrombotic and anti-inflammatory mechanism. Thromb Haemost 115, 835-843, doi:10.1160/TH15-08-0631 (2016).

24. Cherpokova, D.et al. SLAP/SLAP2 prevent excessive platelet (hem)ITAM signaling in thrombosis and ischemic stroke in mice. Blood 125, 185-194, doi:10.1182/blood-2014-06-580597 (2015).

25. Eisinger, F., Patzelt, J. \& Langer, H. F. The Platelet Response to Tissue Injury. Front Med (Lausanne) 5, 317, doi:10.3389/fmed.2018.00317 (2018).

26. Pham, M.et al. Sustained reperfusion after blockade of glycoprotein-receptor-lb in focal cerebral ischemia: an MRI study at 17.6 Tesla. PLoS One 6, e18386, doi:10.1371/journal.pone.0018386 (2011).

27. Schuhmann, M. K.et al. Platelets and lymphocytes drive progressive penumbral tissue loss during middle cerebral artery occlusion in mice. J Neuroinflammation 18,46 , doi:10.1186/s12974-02102095-1 (2021). 
28. Stoll, G. \& Nieswandt, B. Thrombo-inflammation in acute ischaemic stroke - implications for treatment. Nat Rev Neurol 15, 473-481, doi:10.1038/s41582-019-0221-1 (2019).

29. Nour, M., Scalzo, F. \& Liebeskind, D. S. Ischemia-reperfusion injury in stroke. Interv Neurol 1, 185-199, doi:10.1159/000353125 (2013).

30. del Zoppo, G. J. \& Mabuchi, T. Cerebral microvessel responses to focal ischemia. J Cereb Blood Flow Metab 23, 879-894, doi:10.1097/01.WCB.0000078322.96027.78 (2003).

31. El Amki, M. \& Wegener, S. Improving Cerebral Blood Flow after Arterial Recanalization: A Novel Therapeutic Strategy in Stroke. Int J Mol Sci 18, doi:10.3390/ijms18122669 (2017).

32. El Amki, M.et al. Neutrophils Obstructing Brain Capillaries Are a Major Cause of No-Reflow in Ischemic Stroke. Cell Rep 33, 108260, doi:10.1016/j.celrep.2020.108260 (2020).

33. del Zoppo, G. J., Schmid-Schonbein, G. W., Mori, E., Copeland, B. R. \& Chang, C. M. Polymorphonuclear leukocytes occlude capillaries following middle cerebral artery occlusion and reperfusion in baboons. Stroke 22, 1276-1283, doi:10.1161/01.str.22.10.1276 (1991).

\section{Figures}


A

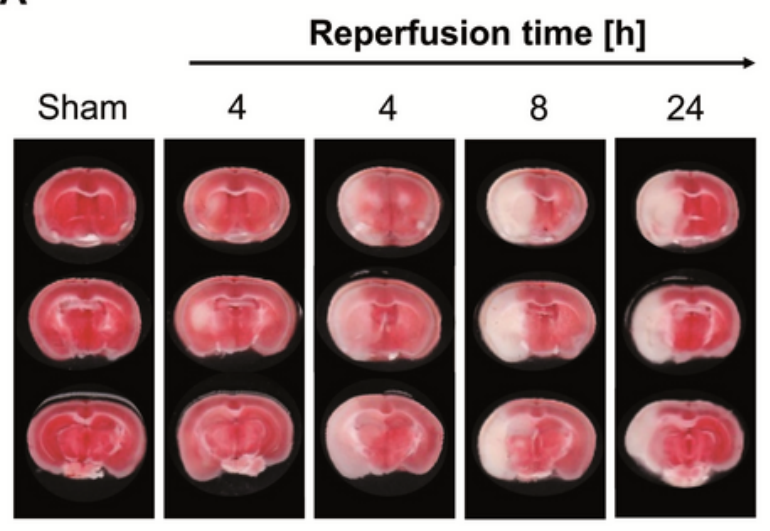

C

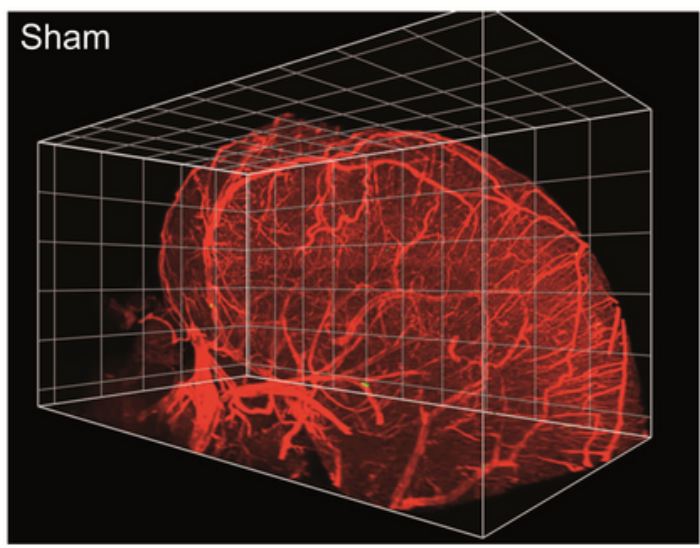

E

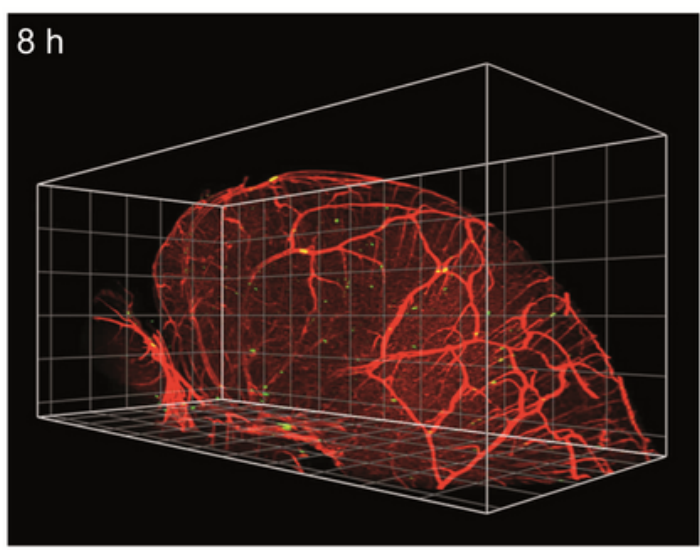

B

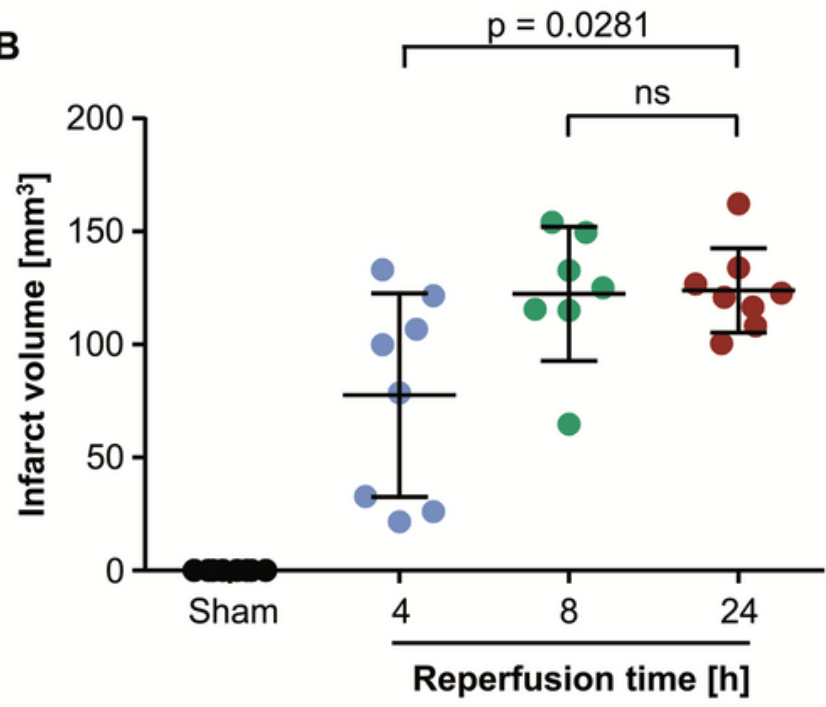

D

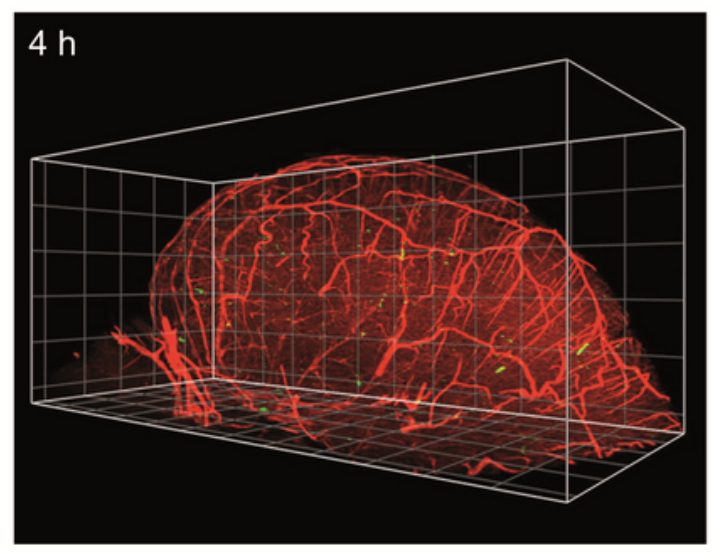

F

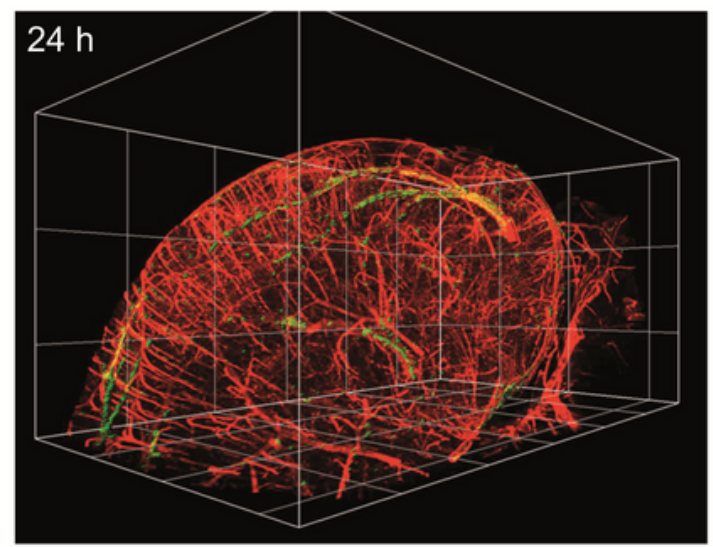

\section{Figure 1}

Cerebral thrombus formation occurs after development of infarcts following tMCAO. (A) Representative images of brain sections stained with TTC to visualize infarcts. Each timepoint shows three consecutive brain sections from one mouse. White: Infarct, Red: viable tissue. (B) Edema corrected quantification of infarct sizes. Data is plotted using GraphPad Prism 7.05 (https://www.graphpad.com) depicted as mean $\pm \mathrm{SD}$ and each dot represents one mouse. $\mathrm{N}=7-8$ per time point. (C-F) 3D reconstruction of the ipsilateral 
hemispheres of mice after (C) sham surgery, (D) $4 \mathrm{~h}$ of reperfusion, (E) $8 \mathrm{~h}$ of reperfusion or (F) $24 \mathrm{~h}$ of reperfusion. Vessels (CD105/CD31; red), platelets (GPIX; green). 3D reconstructions were generated using Biptlane Imaris 9.6 (https://imaris.oxinst.com/); grid size: $0.5 \mathrm{~mm}$. Statistical differences were analyzed using two-tailed Mann-Whitney U test. P-values $<0.05$ were considered statistically significant. Compared to sham, differences to all other groups are highly significant with $p<0.001$.

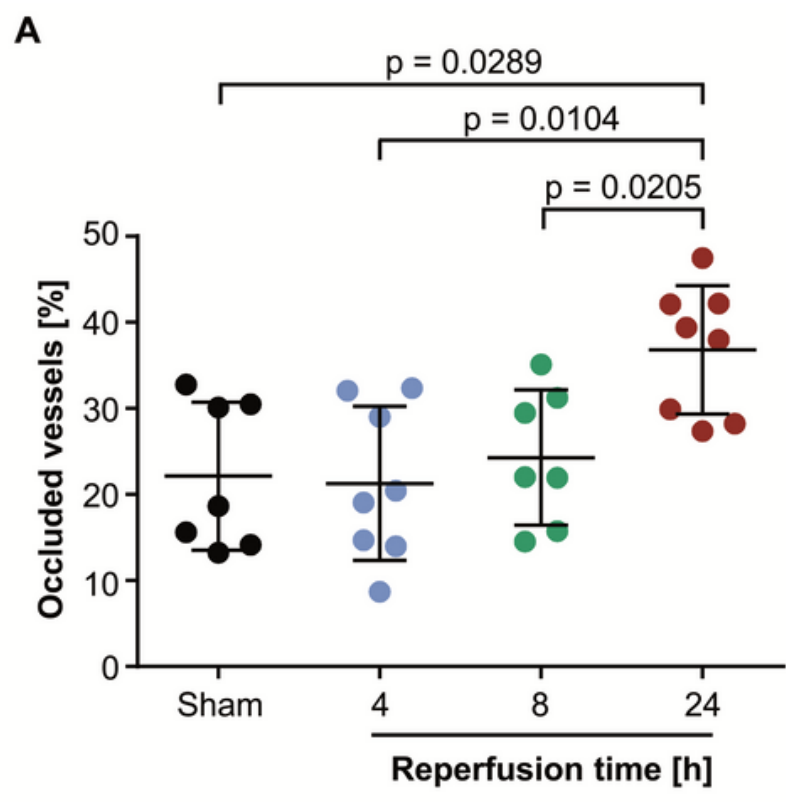

B
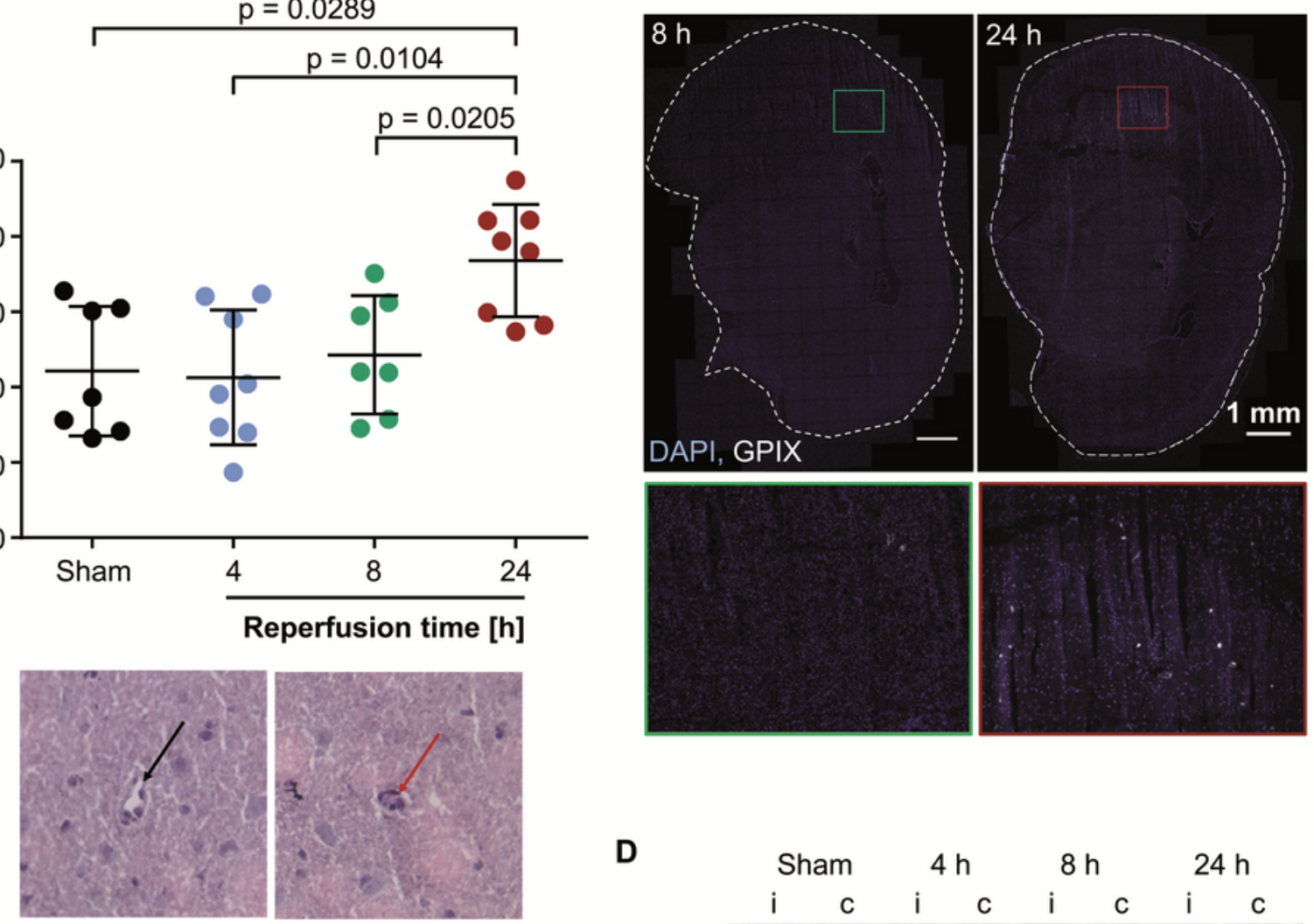

D

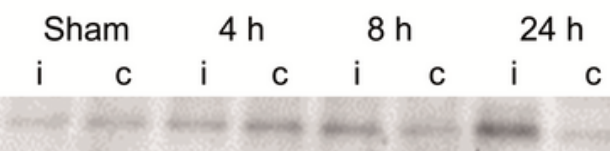

GPIb $\beta$

Actin

C
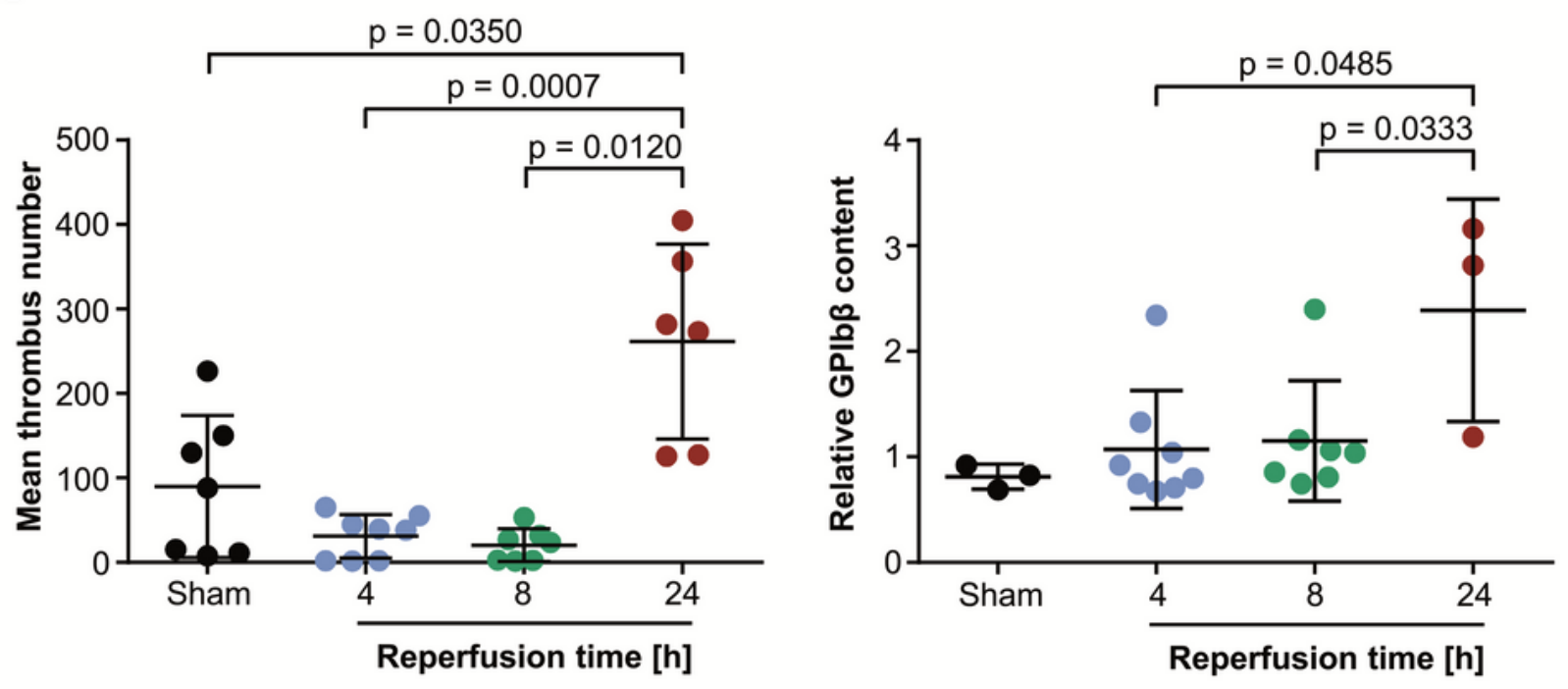

Figure 2 
Cerebral thrombi are barely detectable within the first $8 \mathrm{~h}$ of reperfusion. (A) Quantification of occluded vessels in hematoxylin and eosin stained cryosections. Each dot represents the mean of 3-4 sections from one mouse. N=7-8 per time point. Exemplary image of open (black arrow) and occluded (red arrow) vessel at 20x magnification. (B) Representative z-projections of brain cryosections stained with anti-GPIX (platelets, white) and DAPI (cell nuclei, blue). Lower images show magnification of the respective rectangle in the brain section. (C) Quantification of thrombus number within brain cryosections. Each dot represents the mean of 3-4 sections from one mouse. $N=6-8$ (D) Quantification of platelet GPIb $\beta$ protein $(22 \mathrm{kDa})$ in brain lysates of the cortex. GPIb $\beta$ was normalized to actin (42 kDa) and is indicated as relative content. $\mathrm{N}=3-8$. I: ipsilateral; c: contralateral. Uncropped membrane can be found in Suppl. Fig. 1. Statistical differences were analyzed using two-tailed Mann Whitney $U$ test. P-values $<0.05$ were considered statistically significant and are indicated in the figures. In all panels, data is plotted using GraphPad Prism 7.05 (https://www.graphpad.com) and depicted as mean \pm SD, each dot represents one mouse.

\section{Supplementary Files}

This is a list of supplementary files associated with this preprint. Click to download.

- Gbetalsupplement.pdf

- Supplementaryvideo1sham.mp4

- Supplementaryvideo24h.mp4

- Supplementaryvideo38h.mp4

- Supplementaryvideo424h.mp4 